\title{
Changes in Surface Markers of Human Mesenchymal Stem Cells During the Chondrogenic Differentiation and Dedifferentiation Processes In Vitro
}

\author{
Hyun Jung Lee, ${ }^{1}$ Byung Hyune Choi, ${ }^{2}$ Byoung-Hyun Min, ${ }^{1}$ and So Ra Park ${ }^{2}$
}

\begin{abstract}
Objective. To investigate surface markers showing specific changes during the chondrogenic differentiation and dedifferentiation of human mesenchymal stem cells (MSCs).

Methods. Human MSCs from adult bone marrow were subjected to chondrogenic differentiation in 3-dimensional (3-D) alginate culture with or without transforming growth factor $\beta 3$ (TGFß3) for 2 weeks, followed by dedifferentiation in monolayer for 1 week. Surface antigens were selected from those previously reported to show changes in expression during dedifferentiation of human articular chondrocytes (HACs).

Results. Flow cytometry was used to identify 3 groups of surface antigens with differential expression patterns that were quite different from those previously reported on HACs. Two groups of antigens were expressed at high levels on human MSCs. The expression of the first group of antigens (CD44, CD58, CD81, CD90, CD105, and CD166) was decreased reversibly by the 3-D alginate culture and irreversibly in the presence of TGF B3, except for CD81, which showed reversible changes regardless of TGF $\beta 3$. The expression of the second group of antigens (CD49c, CD49e, and CD151) was decreased during chondrogenic differentiation only
\end{abstract}

Supported by the Ministry for Health, Welfare, and Family Affairs, the Korea Health 21 Research and Development Project (grant A040003), and by the Foundation of the Ministry of Knowledge Economy, Republic of Korea (grant 10024156).

${ }^{1}$ Hyun Jung Lee, PhD, Byoung-Hyun Min, MD, PhD: Ajou University, Suwon, South Korea; ${ }^{2}$ Byung Hyune Choi, $\mathrm{PhD}$, So Ra Park, MD, PhD: Inha University College of Medicine, Incheon, South Korea.

Address correspondence and reprint requests to ByoungHyun Min, MD, PhD, Department of Orthopaedic Surgery, School of Medicine, Ajou University, Wonchon-dong, Youngtong-gu, Suwon 443-749, South Korea (e-mail: bhmin@ajou.ac.kr); or to So Ra Park, $\mathrm{MD}, \mathrm{PhD}$, Department of Physiology, Inha University College of Medicine, 7-206, Sinheung-dong-3-ga, Joong-gu, Incheon 400-712, South Korea (e-mail: srpark@inha.ac.kr).

Submitted for publication May 29, 2008; accepted in revised form April 18, 2009. in the presence of TGF $\beta 3$. During all experimental stages, the expression of the third group of antigens (CD14, CD26, CD49f, CD54, CD106, CD119, and CD140a) was maintained at low levels (expressed on $<30 \%$ of cells), although with some fluctuations.

Conclusion. We speculate that the second group of surface antigens could be negative markers for chondrogenic differentiation of human MSCs.

Mesenchymal stem cells (MSCs) have been widely studied as an alternative cell source for cartilage tissue engineering by virtue of their ability to differentiate into chondrogenic lineage and their phenotypic stability maintained for multiple passages (1-5). Most of the studies until now have focused on finding efficient chondrogenic conditions, such as a 3-dimensional (3-D) environment, growth factors, and/or mechanical forms of stimulation (6-10). Evaluation of the chondrogenic differentiation of MSCs has mainly depended on the expression of chondrocyte phenotypes such as SOX9, type II collagen, and proteoglycans. However, only a little is known about the molecular mechanisms or specific changes in gene expression patterns during the process (10-12), and limited information is available about changes in surface markers (only for CD90 and CD105) during the differentiation of MSCs (13).

Extensive analyses have identified surface markers of MSCs from rats, mice, and humans (14-17). Human MSCs are suggested to be positive for CD44, CD90, and CD105 and negative for CD14, CD34, and CD45 (17). However, their expression pattern was shown to change depending on the culture conditions or specific types of stimulation $(13,18,19)$. Recently, DiazRomero et al reported on the expression patterns of 52 surface antigens on human articular chondrocytes (HACs) during the dedifferentiation process in monolayer culture for 2 weeks (20). They identified a subset of surface markers expressed on HACs and suggested that 
A

\begin{tabular}{|ccc|}
\multicolumn{1}{c}{ I } & II & III \\
$\begin{array}{c}\text { Proliferation stage } \\
\text { (3 passages) }\end{array}$ & $\begin{array}{c}\text { Differentiation stage } \\
\text { (2 weeks) }\end{array}$ & $\begin{array}{c}\text { Dedifferentiation stage } \\
\text { (1 week) }\end{array}$ \\
\hline Monolayer culture & 3-D Alginate culture & Monolayer culture \\
\hline
\end{tabular}

B

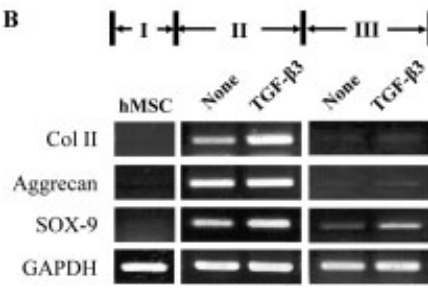
C

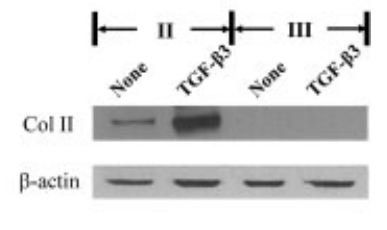

Figure 1. The experimental plan. A, Human mesenchymal stem cells (MSCs; [hMSC]) were subjected to 3 experimental steps for cell proliferation (I), chondrogenic differentiation (II), and dedifferentiation (III). Human MSCs were subjected to chondrogenic differentiation in 3-dimensional (3-D) alginate culture with or without (None) transforming growth factor $\beta 3$ (TGF $\beta 3$ ) for 2 weeks. After alginate culture, recovered cells were then cultured as monolayer for 1 week to induce dedifferentiation of cells. B, During the experimental stages, expression of chondrogenic markers (type II collagen [Col II], aggrecan, and SOX9) was examined by reverse transcriptase-polymerase chain reaction analysis. $\mathbf{C}$, The protein level of type II collagen during the differentiation and dedifferentiation stages was examined by Western blot analysis, with $\beta$-actin used as an internal control.

HACs had reverted to a primitive stem cell phenotype after dedifferentiation based on the induced expression of some human MSC markers, such as CD10, CD90, CD105, and CD166. The expression of some surface antigens, such as CD14, CD49a, CD54, and CD106, was also shown to be reduced along with the dedifferentiation process in their experiment. It is not clear, however, if these antigens can be regarded as specific markers for active chondrocytes or for the chondrogenic differentiation of stem cells.

In the present study, we investigated changes in the expression of surface antigens on human MSCs during the chondrogenic differentiation and dedifferentiation processes in order to enhance the understanding of phenotypic changes during these processes and to identify specific markers for differentiated chondrocytes. Human MSCs from the bone marrow were subjected to the following 3 conditions: 1) expansion in monolayer culture until passage 3,2 ) chondrogenic differentiation in 3-D alginate culture for 2 weeks, and 3) dedifferentiation in monolayer culture for 1 week (Figure 1A). The expression pattern of selected surface markers reported by Diaz-Romero et al (20) was examined during these processes.

\section{MATERIALS AND METHODS}

Isolation and culture of human bone marrow MSCs. With informed consent, bone marrow samples were obtained from trabecular bones of 3 hematologically normal patients undergoing routine total hip replacement surgery. The patients had a mean age of 64 years (range 55-73 years). Primary culture of bone marrow stromal cells was performed as previously described (21). Briefly, marrow cells were isolated from trabecular bone marrow samples in Dulbecco's phosphate buffered saline (PBS) (Gibco BRL, Carlsbad, CA) and harvested by centrifugation at $500 \mathrm{~g}$ for 5 minutes at room temperature. The cell pellet was resuspended in $10 \mathrm{ml} \alpha$-minimum essential medium ( $\alpha$-MEM) supplemented with $10 \%$ fetal bovine serum (FBS) and passed through nylon mesh $(90-\mu \mathrm{m}$ pore size; Lockertex, Warrington, UK). Aliquots of cell suspensions were diluted with $0.4 \%$ (weight/volume) trypan blue, and the number and viability of cells were determined. Cells $\left(1.5 \times 10^{7}\right)$ were plated on a $150-\mathrm{mm}$ culture plate and incubated at $37^{\circ} \mathrm{C}$ in a $5 \% \mathrm{CO}_{2}$ atmosphere. After 6 days, nonadherent hematopoietic cells were removed, and the MSCs on the culture plate were replenished with fresh medium supplemented with $10 \%$ FBS. The medium was changed twice per week thereafter. The cells were expanded in a monolayer culture until passage 3 with an interval of $\sim 1-2$ weeks for each passage, after which they were moved to a 3-D culture for chondrogenic differentiation.

Chondrogenic differentiation of human MSCs in alginate layer culture. The alginate layer culture of human MSCs was performed as described previously $(9,22)$. Human MSCs were suspended in $2 \%$ alginate (Sigma, St. Louis, MO) solution at a density of $2 \times 10^{6} / \mathrm{ml}$. The cell/alginate mixture was added slowly in a 12-well Transwell insert and spread evenly over the surface. The Transwell was then immersed in $1 \mathrm{ml}$ of sterile $102 \mathrm{mM} \mathrm{CaCl}$ for 10 minutes. The human $\mathrm{MSC} /$ alginate layer was washed twice with $0.15 \mathrm{M} \mathrm{NaCl}$ for 10 minutes each and once with serum-free chondrogenic defined medium containing high-glucose Dulbecco's modified Eagle's medium (DMEM), ITS supplement $(1.0 \mathrm{mg} / \mathrm{ml}$ insulin from bovine pancreas, $0.55 \mathrm{mg} / \mathrm{ml}$ human transferrin, and $0.5 \mu \mathrm{g} / \mathrm{ml}$ sodium selenite), $50 \mu \mathrm{g} / \mathrm{ml} \mathrm{L}$-ascorbic acid 2-phosphate, 100 $\mathrm{n} M$ dexamethasone, $40 \mu \mathrm{g} / \mathrm{ml}$ proline, $1.25 \mathrm{mg} / \mathrm{ml}$ bovine serum albumin, and $100 \mu \mathrm{g} / \mathrm{ml}$ sodium pyruvate (all from Sigma) (23). The human MSC/alginate layers were then moved to $35-\mathrm{mm}$ culture dishes and overlaid with serum-free chondrogenic defined medium. The samples were cultured at $37^{\circ} \mathrm{C}$ in a $5 \% \mathrm{CO}_{2}$ atmosphere for 2 weeks for chondrogenic differentiation, with or without treatment with transforming growth factor $\beta 3$ (TGF $\beta 3$ ). Samples were treated with $10 \mathrm{ng} / \mathrm{ml}$ TGF $\beta 3$ (R\&D Systems, Minneapolis, MN) at the time of media change.

Dedifferentiation of differentiated cells in a monolayer culture. After chondrogenic differentiation for 2 weeks, the alginate layer cultures were washed twice with $0.15 \mathrm{M} \mathrm{NaCl}$ and incubated in 3 volumes of $55 \mathrm{~m} M$ sodium citrate solution at $37^{\circ} \mathrm{C}$ for 15 minutes to dissolve the layer. The cells recovered were harvested by centrifugation at $1,500 \mathrm{~g}$ for 5 minutes and were replated on a monolayer culture in high-glucose DMEM supplemented with $10 \%$ FBS for 7 days.

Reverse transcriptase-polymerase chain reaction (RTPCR). Total RNA was extracted from cells using TRIzol reagent (Invitrogen, Carlsbad, CA). For the alginate culture, 
cells were first recovered from the alginate layer as described above. A total of $1 \mu \mathrm{g}$ RNA was used for the first-strand complementary DNA (cDNA) synthesis using the Superscript First Strand Synthesis System (Roche, Indianapolis, IN), and 2 $\mu \mathrm{g}$ of the synthesized cDNA was used for PCR. Sequences of specific primers were as follows: SOX9, 5'. CACACAGCTCACTCGACCTTG-3' (sense) and 5' TTCGGTTATTTTTAGGATCATCTCG-3' (antisense); type II collagen, 5'-GATATTGCACCTTTGGACAT-3' (sense) and 5'-CCCACAATTTAAGCAAGAAG-3' (antisense); and aggrecan, 5'-GAAAGGTGTTGTGTTCCACT-3' (sense) and 5'-GTCATAGGTCTCGTTGGTGT-3' (antisense). GAPDH (5'-GGACATGAGTCCTTCCACGAT-3' [sense] and 5'GGTGAAGGTCGGAGTCAACGG-3' [antisense]) was used as an internal control. To exclude the possibility of genomic DNA contamination, RT-PCR was also carried out using total RNA without RT.

Western blot analysis. Cell extracts were prepared using a lysis buffer $(40 \mathrm{~m} M$ Tris $\mathrm{HCl}[\mathrm{pH}$ 8.0], $120 \mathrm{~m} M \mathrm{NaCl}$, $0.5 \%$ Nonidet P40, $100 \mu \mathrm{g} / \mathrm{ml}$ phenylmethylsulfonyl fluoride, and $2 \mu \mathrm{g} / \mathrm{ml}$ each of aprotinin, pepstatin, and leupeptin). The total amount of proteins was determined using the bicinchoninic acid assay (Sigma). The proteins were separated on an $8 \%$ sodium dodecyl sulfate polyacrylamide gel and transferred onto nitrocellulose membranes (Protran; Schleicher \& Schuell, Keene, NH). After blocking, the blots were incubated overnight at $4^{\circ} \mathrm{C}$ with primary antibodies to type II collagen (Chemicon, Temecula, CA). The blots were then incubated with horseradish peroxidase-conjugated goat anti-mouse IgG for 1 hour at room temperature. Immunoreactive signals were detected using an enhanced chemiluminescence kit (Amersham, Piscataway, NJ).

Flow cytometry. Cells recovered from monolayer or alginate culture were washed twice in PBS. Single-cell suspensions in PBS $\left(1 \times 10^{6}\right.$ cells $\left./ 20 \mu \mathrm{l}\right)$ were stained at $4^{\circ} \mathrm{C}$ for 40 minutes with antibodies to CD14, CD26, CD44, CD49c, CD49e, CD49f, CD54, CD58, CD81, CD90, CD105, CD106, CD119, CD140a, CD151, or CD166 according to the manufacturers' instructions. Isotype IgG was used as a negative control. All antibodies were purchased from BD PharMingen (San Jose, CA) except for CD105, which was purchased from Ancell (Bayport, MN). Detailed information about the antibodies we used is available on our Web site (www.cellntissue. com). The cells were then washed 3 times with $0.5 \mathrm{ml}$ PBS and resuspended in $0.5 \mathrm{ml} 2 \%$ paraformaldehyde in PBS for fixing. The cells were subjected to flow cytometry using a FACScan instrument and CellQuest software (Becton Dickinson Immunocytometry Systems, San Jose, CA).

\section{RESULTS}

Chondrogenic differentiation and dedifferentiation of human MSCs. The overall experimental plan of the study is illustrated in Figure 1A. The experiments were performed independently using MSCs from 3 donors in the whole study. MSCs from each donor showed a similar capacity for cell proliferation and chondrogenic differentiation. In the proliferation stage, MSCs from human bone marrow were expanded for 3 passages in order to yield a sufficient amount of cells with well-maintained stem cell phenotypes. Chondrogenic differentiation of human MSCs was then induced for 2 weeks using a 3-D alginate culture system in chondrogenic defined medium, with or without TGF $\beta 3$. Finally, the dedifferentiation stage was carried out by culturing the differentiated cells on a monolayer for 1 week in proliferation medium supplemented with $10 \%$ FBS.

When examined by RT-PCR analysis, human MSCs in an initial monolayer culture were negative for the expression of chondrogenic markers such as type II collagen, aggrecan, and SOX9 (Figure 1B). The expression of these chondrogenic markers was increased by 3-D alginate culture of human MSCs. It was significantly higher than that induced by treatment with TGF $\beta 3$. We speculate that 3-D alginate culture in defined medium without TGF $\beta 3$ was not sufficient to fully develop hyaline cartilage-like constructs with lacunae formation, as shown in our previous study using rabbit MSCs (9). After reculturing the differentiated cells on a monolayer for 1 week, the expression of chondrogenic marker genes was dramatically reduced in all experimental groups, showing that the cells had dedifferentiated and lost their chondrogenic phenotypes. The chondrogenic differentiation and dedifferentiation of human MSCs were also confirmed by Western blotting analysis for type II collagen (Figure 1C).

Effect of TGF $\beta 3$ treatment on the expression of selected sets of surface antigens. We analyzed the effect of TGF $\beta 3$ treatment on the expression of selected surface antigens that had shown changes in expression on HACs during the dedifferentiation process in the study by Diaz-Romero et al (20). Surface antigens were categorized into 3 groups as described by Diaz-Romero et al: 1) tetraspanins, ectoenzymes, and other miscellaneous surface antigens (CD26, CD81, CD90, and CD151); 2) cell adhesion molecules including integrins (CD44, CD49c, CD49e, CD49f, CD54, CD58, CD106, and CD166); and 3) receptors for growth factors or other ligands (CD14, CD105, CD119, and CD140a).

Among the first group of antigens, human MSCs were positive for CD90 (Thy-1), CD81, and CD151 (Figure 2). However, they all showed quite different and diverse expression patterns during the differentiation and dedifferentiation processes. The expression of CD90 was decreased reversibly by the 3-D alginate culture alone but was reduced irreversibly in the presence of TGF $\beta 3$. The expression of CD 81 was decreased reversibly by the $3-\mathrm{D}$ alginate culture both in the absence and in the presence of TGF $\beta 3$. The expression of CD151 was 


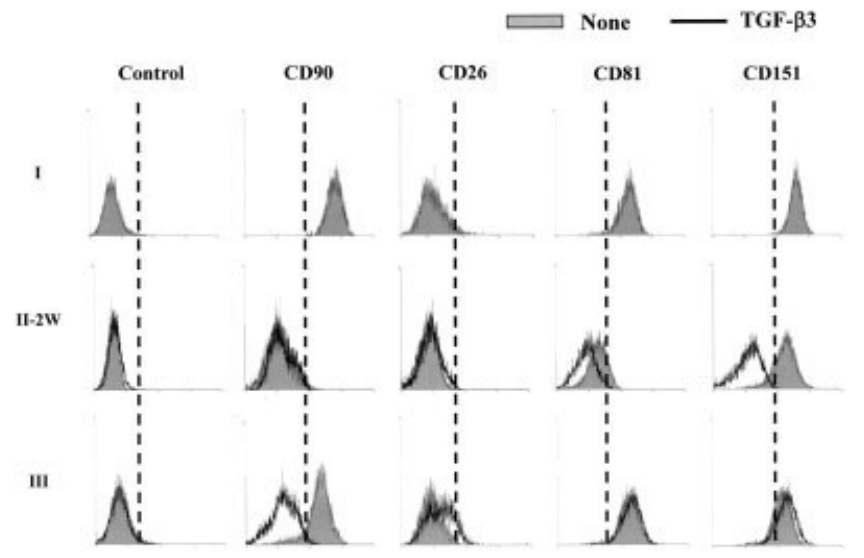

Figure 2. Effects of TGF $\beta 3$ treatment on the expression of tetraspanins, ectoenzymes, and other miscellaneous surface antigens. Under the experimental plan shown in Figure 1, the effect of treatment with TGF $\beta 3$ on the expression of an ectoenzyme (CD26), tetraspanins (CD81 and CD151), and a miscellaneous surface marker (CD90) was examined by flow cytometry. Analysis was carried out at passage 3 of monolayer culture (I), at 2 weeks of 3-D alginate culture (II-2W), and at 1 week of replating (III). Vertical dashed lines, based on stage I, serve as the baseline for comparisons with other groups. See Figure 1 for definitions.

not significantly changed without TGF $\beta 3$ but was reduced reversibly by TGF $\beta 3$ treatment during the 3-D alginate culture. The expression of CD26 (peptidase IV) was not changed significantly under any condition.

Among the integrins tested, human MSCs were

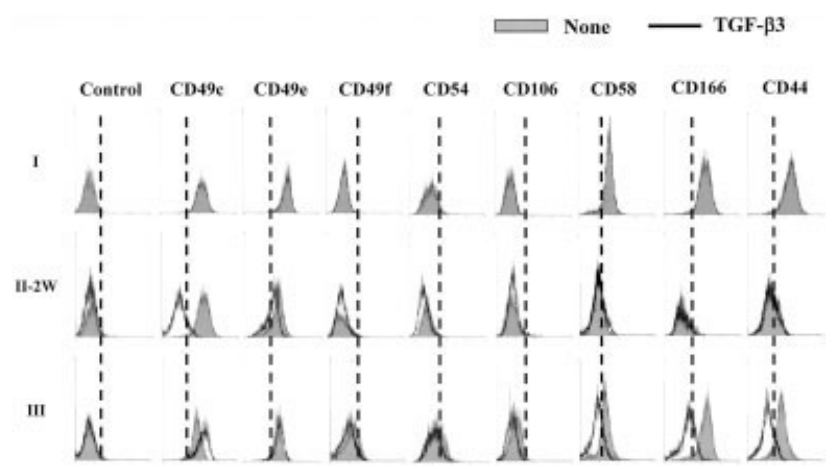

Figure 3. Effects of TGF $\beta 3$ treatment on the expression of adhesion molecules. Under the experimental plan shown in Figure 1, the effect of treatment with TGF $\beta 3$ on the expression of integrins (CD 49c, CD49e, and CD49f), immunoglobulins (CD54 [intercellular adhesion molecule 1], CD106 [vascular cell adhesion molecule 1], CD58 [lymphocyte function-associated antigen 3], and CD166 [activated leukocyte cell adhesion molecule]), and other adhesion molecules (CD44) was examined by flow cytometry. Analysis was carried out at passage 3 of monolayer culture (I), at 2 weeks of 3-D alginate culture (II-2W), and at 1 week of replating (III). Vertical dashed lines, based on stage I, serve as the baseline for comparisons with other groups. See Figure 1 for definitions.

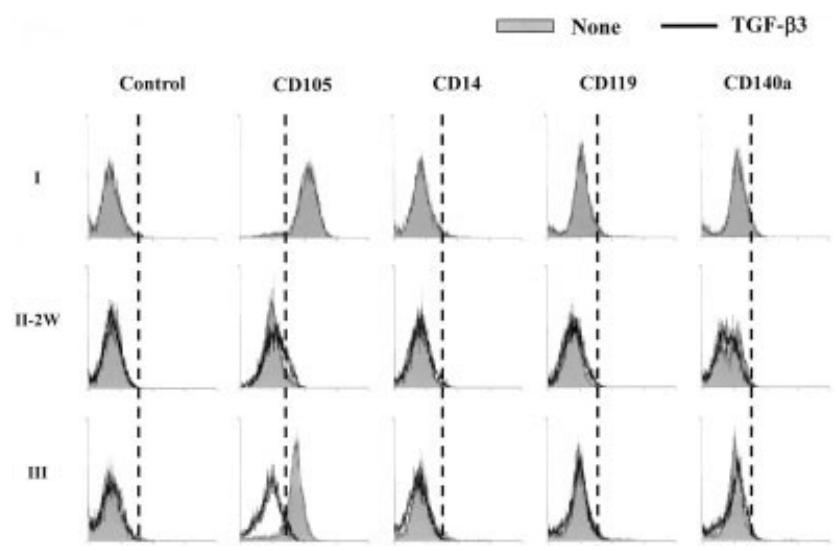

Figure 4. Effects of TGF $\beta 3$ treatment on the expression of cell surface receptors. Under the experimental plan shown in Figure 1, the effect of treatment with TGF $\beta 3$ on the expression of cytokine or lipopolysaccharide receptors (CD14, CD105, CD119, and CD140a) was examined by flow cytometry. Analysis was carried out at passage 3 of monolayer culture (I), at 2 weeks of 3-D alginate culture (II-2W), and at 1 week of replating (III). Vertical dashed lines, based on stage I, serve as the baseline for comparisons with other groups. See Figure 1 for definitions.

positive for $\mathrm{CD} 49 \mathrm{c}$ ( $\alpha 3$ integrin) and $\mathrm{CD} 49 \mathrm{e}(\alpha 5$ integrin), but negative for CD49f ( $\alpha 6$ integrin) (Figure 3). The expression of these integrins was not further changed by the 3-D alginate culture alone, and only the expression of CD $49 \mathrm{c}$ was decreased reversibly by TGF $\beta 3$ treatment. Among the other cell adhesion molecules tested, human MSCs were positive for CD44 (hyaluronan receptor), CD58 (lymphocyte function-associated antigen 3), and CD166 (activated leukocyte cell adhesion molecule) and negative for CD54 (intercellular adhesion molecule 1) and CD106 (vascular cell adhesion molecule 1). The expression of CD44, CD58, and CD166 was decreased reversibly by the $3-\mathrm{D}$ alginate culture alone and irreversibly in the presence of TGF $\beta 3$. The expression of CD54 and CD106 was not observed under any condition.

In the case of cell surface receptors, human MSCs were positive only for CD105 (endoglin) and negative for the other surface antigens (Figure 4). The expression of CD105 was decreased reversibly by the 3-D alginate culture but irreversibly in the presence of TGF $\beta 3$. The expression of CD14 (lipopolysaccharide receptor), CD119 (interferon- $\gamma$ receptor), and CD140a (platelet-derived growth factor receptor $\alpha$ ) was not detected at significant levels under any condition.

The results of flow cytometric analyses are summarized in Table 1. The surface antigens were classified into 3 groups based on their changes in expression in 3-D alginate culture in the presence or absence of 
Table 1. Summary of the results of flow cytometry*

\begin{tabular}{|c|c|c|c|c|c|c|c|}
\hline \multirow[b]{3}{*}{$\begin{array}{c}\text { Group, } \\
\text { surface antigen }\end{array}$} & \multicolumn{5}{|c|}{ Human MSCs } & & \\
\hline & \multirow[b]{2}{*}{$\begin{array}{l}\text { Monolayer } \\
\text { culture } \\
\text { (3 passages) }\end{array}$} & \multicolumn{2}{|c|}{$\begin{array}{c}\text { No TGF } \beta 3 \text { treatment during } \\
\text { alginate culture }\end{array}$} & \multicolumn{2}{|c|}{$\begin{array}{l}\text { TGF } \beta 3 \text { treatment during } \\
\text { alginate culture }\end{array}$} & \multicolumn{2}{|c|}{$\begin{array}{c}\text { Human chondrocytes } \\
\text { (Diaz-Romero et al, 2005) }\end{array}$} \\
\hline & & $\begin{array}{l}\text { Alginate } \\
\text { culture } \\
(2 \text { weeks })\end{array}$ & $\begin{array}{l}\text { Monolayer } \\
\text { culture } \\
\text { (7 days) }\end{array}$ & $\begin{array}{l}\text { Alginate } \\
\text { culture } \\
(2 \text { weeks })\end{array}$ & $\begin{array}{l}\text { Monolayer } \\
\text { culture } \\
\text { (7 days) }\end{array}$ & $\begin{array}{l}\text { Monolayer } \\
\text { culture } \\
\text { ( } 24 \text { hours) }\end{array}$ & $\begin{array}{l}\text { Monolayer } \\
\text { culture } \\
(2 \text { weeks })\end{array}$ \\
\hline \multicolumn{8}{|l|}{ Group 1} \\
\hline CD44 & $98.52 \pm 3.24$ & $48.14 \pm 8.42$ & $98.72 \pm 2.43$ & $42.71 \pm 7.35$ & $36.25 \pm 9.52$ & $97.61 \pm 0.68$ & $91.80 \pm 6.95$ \\
\hline CD58 & $72.51 \pm 23.52$ & $12.42 \pm 1.23$ & $58.46 \pm 4.38$ & $16.52 \pm 3.56$ & $14.18 \pm 8.52$ & $2.50 \dagger$ & $4.10 \dagger$ \\
\hline CD81キ & $99.75 \pm 0.27$ & $43.58 \pm 8.23$ & $95.84 \pm 0.85$ & $21.53 \pm 6.95$ & $97.22 \pm 0.39$ & $93.98 \pm 3.97$ & $84.55 \pm 8.83$ \\
\hline CD90 & $99.97 \pm 0.03$ & $18.67 \pm 2.54$ & $99.56 \pm 0.59$ & $21.55 \pm 9.42$ & $25.64 \pm 5.96$ & $3.18 \pm 1.19$ & $99.88 \pm 0.03$ \\
\hline CD105 & $97.26 \pm 4.19$ & $10.23 \pm 8.91$ & $90.79 \pm 2.78$ & $11.17 \pm 9.31$ & $10.71 \pm 2.91$ & $67.35 \pm 4.26$ & $95.00 \pm 4.12$ \\
\hline CD166 & $99.68 \pm 0.21$ & $26.41 \pm 8.54$ & $98.79 \pm 2.48$ & $22.41 \pm 10.38$ & $41.63 \pm 8.62$ & $35.21 \pm 6.62$ & $92.31 \pm 5.53$ \\
\hline \multicolumn{8}{|l|}{ Group 2} \\
\hline $\mathrm{CD} 49 \mathrm{c}$ & $99.57 \pm 0.31$ & $97.25 \pm 3.62$ & $74.33 \pm 4.36$ & $12.36 \pm 6.93$ & $93.52 \pm 5.30$ & $66.59 \pm 4.56$ & $84.06 \pm 11.35$ \\
\hline $\mathrm{CD} 49 \mathrm{e}$ & $99.92 \pm 0.01$ & $97.26 \pm 0.30$ & $97.21 \pm 1.84$ & $57.72 \pm 13.56$ & $98.33 \pm 0.71$ & $94.24 \pm 3.74$ & $99.91 \pm 0.02$ \\
\hline CD151 & $99.85 \pm 0.12$ & $98.39 \pm 7.23$ & $94.36 \pm 3.65$ & $32.88 \pm 7.85$ & $98.54 \pm 0.63$ & $94.00 \pm 3.96$ & $84.56 \pm 8.83$ \\
\hline \multicolumn{8}{|l|}{ Group 3} \\
\hline CD14 & $8.89 \pm 5.49$ & $2.49 \pm 1.60$ & $5.28 \pm 1.41$ & $1.73 \pm 0.74$ & $2.68 \pm 0.88$ & $49.94 \pm 4.15$ & $4.39 \pm 1.33$ \\
\hline $\mathrm{CD} 26$ & $14.35 \pm 5.23$ & $3.27 \pm 1.09$ & $7.54 \pm 0.32$ & $3.56 \pm 3.06$ & $39.56 \pm 17.21$ & $3.01 \dagger$ & $4.85 \dagger$ \\
\hline CD49f & $8.82 \pm 1.82$ & $10.28 \pm 1.62$ & $2.75 \pm 1.47$ & $0.32 \pm 0.13$ & $5.87 \pm 2.14$ & $11.77 \pm 2.26$ & $60.78 \pm 8.00$ \\
\hline CD54 & $28.62 \pm 18.53$ & $12.21 \pm 7.54$ & $35.47 \pm 8.16$ & $5.56 \pm 0.23$ & $22.35 \pm 3.58$ & $88.55 \pm 7.33$ & $78.41 \pm 4.62$ \\
\hline CD106 & $8.76 \pm 1.28$ & $12.32 \pm 2.23$ & $9.14 \pm 0.26$ & $0.62 \pm 0.14$ & $7.28 \pm 0.42$ & $95.31 \pm 2.49$ & $62.01 \pm 6.80$ \\
\hline CD119 & $13.56 \pm 10.68$ & $2.36 \pm 0.53$ & $8.66 \pm 0.62$ & $3.23 \pm 1.62$ & $5.33 \pm 0.13$ & $2.90 \dagger$ & $3.10 \dagger$ \\
\hline CD140a & $11.98 \pm 1.80$ & $8.61 \pm 1.08$ & $7.39 \pm 0.17$ & $2.24 \pm 1.85$ & $7.49 \pm 2.04$ & $2.40 \dagger$ & $5.60 \dagger$ \\
\hline
\end{tabular}

* Values are the mean \pm SD percentage of cells expressing each surface antigen and are derived from 3 independent experiments using mesenchymal stem cells (MSCs) from individual donors. Results were also compared with those reported by Diaz-Romero et al (20). The surface antigens examined were classified into groups 1,2, and 3 based on their expression patterns during chondrogenic differentiation and dedifferentiation of human MSCs (see Results).

$\dagger$ No data were available for the SD.

¥CD81 showed a somewhat different expression pattern from the other group 1 antigens, in that transforming growth factor $\beta 3$ (TGF $\beta 3$ ) did not affect its expression pattern at the dedifferentiation stage.

TGF $\beta 3$ treatment. The surface antigens in group 1 were all expressed at high levels in human MSCs in monolayer culture; their expression was decreased reversibly by the 3-D alginate culture and irreversibly in the presence of TGF 33 , except for CD81, which showed reversible changes regardless of TGF $\beta 3$. The surface antigens in group 2 were also well expressed in human MSCs in monolayer culture, and their expression decreased reversibly only in the presence of TGF $\beta 3$. The surface antigens in group 3 were expressed at low levels in human MSCs and showed no significant increase in their expression levels during the differentiation and dedifferentiation processes (mostly $<30 \%$ overall).

Early changes in the expression of group 1 surface antigens. The expression of group 1 surface antigens other than CD81 was examined before 3-D alginate culture and on days $1,3,7$, and 14 after 3 -D alginate culture to determine whether the decrease in expression levels seen at 2 weeks was a rapid change or a gradual one. As shown in Figure 5, their expression levels clearly started to decrease from day 1 , but the decrease was gradual with time after 3-D alginate cul-

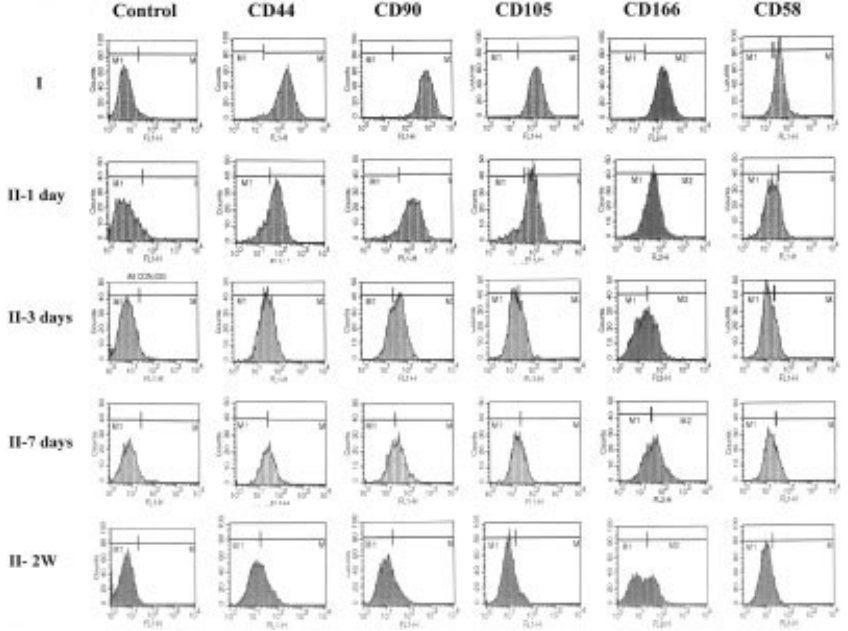

Figure 5. The early expression pattern of group 1 surface markers other than CD81 during the chondrogenic differentiation of human MSCs. Under the experimental plan shown in Figure 1, the expression of CD44, CD90, CD105, CD166, and CD58 was examined before 3-D alginate culture (I) and on days 1, 3, 7, and 14 (II-1 day, II-3 days, II-7 days, and II-2W, respectively) after 3-D alginate culture by flow cytometry. See Figure 1 for definitions. 
ture. This result suggests that the decrease in expression levels was not caused by rapid changes in the culture environment itself and might involve progressive changes in cell physiology. The expression of these surface antigens was also decreased in basal medium ( $\alpha$-MEM, 10\% FBS), which suggests again that changes in their expression patterns were caused by changes in the culture environment but were not specific to chondrogenic differentiation (additional data are available at http://www.cellntissue.com/data/bbs/0905070. 614216.jpg).

\section{DISCUSSION}

Using flow cytometry, we systematically investigated changes in the expression of 16 surface antigens on human MSCs that depended on chondrogenic differentiation and dedifferentiation processes. The surface antigens tested in the present study were chosen based on the previously reported study by Diaz-Romero et al (20), who identified groups of surface antigens specifically expressed in dedifferentiated human chondrocytes. As summarized in Table 1, flow cytometry was used to identify 3 groups of surface antigens showing differential expression patterns during the experimental stages. We could not find any specific marker whose expression was induced by the chondrogenic differentiation of human MSCs, but we did find subsets of surface antigens whose expression was reduced during the process. No specific expression pattern was observed according to the categories of surface antigens described by Diaz-Romero et al (20), suggesting that specific groups of antigens, such as cell adhesion molecules, cell surface receptors, and the like, have no apparent roles in chondrogenic differentiation and dedifferentiation. Specific functions of the surface antigens, particularly in cartilage, have been well summarized by Diaz-Romero et al (20). The expression pattern of the surface markers will be discussed in detail group by group and compared with the results reported by Diaz-Romero et al (20).

The group 1 antigens (CD44, CD58, CD81, CD90, CD105, and CD166) were all well-known surface markers of human MSCs except for CD81 $(17,24)$ and were also expressed at high levels on expanding human MSCs in the present study. Their expression levels decreased during the chondrogenic differentiation process, with or without TGF $\beta 3$, consistent with a previous study showing a similar result for CD44, CD90, and CD105 in micromass culture of human MSCs (13). We speculate that the group 1 surface antigens might be markers of human MSCs for changes in the microenvi- ronment, but not specific negative markers in active chondrocytes. First, their expression level started to decrease right after the 3-D alginate culture without TGF $\beta 3$ treatment and became significantly low on days 7 and 14, when no significant chondrogenic differentiation was observed. Second, the irreversible decrease in their expression induced by TGF $\beta 3$ treatment was inconsistent with their high level of expression in dedifferentiated HACs (20). Further, some of the markers such as CD44, CD58, and CD105 were also shown to be expressed in active HACs $(20,25)$.

The group 2 surface antigens $(C D 49 c, C D 49 e$, and CD151) were also expressed at high levels in human MSCs. Their expression levels showed no significant change at all stages in the absence of TGF $\beta 3$, but decreased reversibly during the differentiation stage only in the presence of TGF $\beta 3$. This result suggests that they could be negative markers, at least for the chondrogenic differentiation of human MSCs. Further analyses are necessary to address this possibility. However, they do not appear to be negative markers for chondrocytes because they were shown to be expressed at high levels in active HACs $(20,25)$. The group 3 surface antigens were expressed at low levels in human MSCs, and their expression levels fluctuated without a significant increase during the differentiation and dedifferentiation processes. Also, the low expression levels of CD54 and CD106 are inconsistent with their high levels of expression both in active and in dedifferentiated HACs (20).

Our results suggest that expression patterns of the surface markers overall were quite different between HACs and human MSCs during differentiation and/or dedifferentiation processes. We speculate that the chondrogenic differentiation condition of human MSCs in vitro is artificial, especially given continuous treatment with TGF $\beta 3$, and that it does not completely recapitulate the dynamic changes seen during chondrogenic development in vivo. There might also be innate differences between MSCs differentiating into chondrocytes in vivo and human MSCs isolated from adult bone marrow in vitro. Therefore, expression patterns of surface antigens in this study may not definitely represent the phenomena in vivo, but these antigens could be specific markers for chondrogenic differentiation of human MSCs in vitro.

Human MSCs isolated from adult bone marrow in this study were positive for CD44, CD49c, CD49e, CD81, CD90, CD105, CD151, and CD166 and were negative for CD14, CD26, CD49f, CD54, CD106, CD119, and CD140a. Most of them are already known 
in human MSCs $(17,24,26)$, but the expression pattern of CD49c was introduced for the first time in the present study. The expression of CD81 both on human MSCs and on chondrocytes has been reported previously, but its function has not yet been identified $(27,28)$. Grogan et al showed that CD44, CD151, and CD49c were expressed at significantly higher levels on chondrocytes with higher chondrogenic capacity (25). However, they were all highly expressed on human MSCs in the present study and in other studies, and it is questionable whether they could be specific markers for active chondrocytes (27). CD58 was expressed at moderate levels on human MSCs in the present study. Independent groups have reported its expression either on normal HACs (20) or, in contrast, only in arthritic joints (29). The expression levels of CD54 and CD106 were shown to be variable depending on the donor ages and passage numbers (26).

Previously, clonal populations of dedifferentiated adult HACs were reported to gain stem cell properties, thus becoming able to differentiate into the chondrogenic, osteogenic, and adipogenic lineages (30). DiazRomero et al also suggested that dedifferentiated HACs had reverted to a primitive phenotype, at least in terms of surface markers, because some MSC markers such as CD10, CD90, CD105, and CD166 were up-regulated after dedifferentiation (20). However, we are not sure that human MSCs, once they have differentiated into the chondrogenic lineage with TGF $\beta 3$ treatment, could regain their stem cell capacity after dedifferentiation, because typical human MSC markers such as CD44, CD90, and CD105 were lost irreversibly during differentiation. The irreversible loss of typical human MSC markers could be an unexpected outcome of long-term treatment with TGF $\beta 3$ in vitro. It will be an interesting project to examine the stem cell phenotype, after dedifferentiation, of human MSCs that have differentiated with TGF $\beta 3$ treatment.

\section{AUTHOR CONTRIBUTIONS}

Drs. Min and Park had full access to all of the data in the study and take responsibility for the integrity of the data and the accuracy of the data analysis.

Study design. Min, Park.

Acquisition of data. Lee.

Analysis and interpretation of data. Lee, Choi.

Manuscript preparation. Lee, Choi.

Statistical analysis. Lee.

\section{REFERENCES}

1. Johnstone B, Hering TM, Caplan AI, Goldberg VM, Yoo JU. In vitro chondrogenesis of bone marrow-derived mesenchymal progenitor cells. Exp Cell Res 1998;238:265-72.
2. Majumdar MK, Banks V, Peluso DP, Morris EA. Isolation, characterization, and chondrogenic potential of human bone marrow-derived multipotential stromal cells. J Cell Physiol 2000; 185:98-106.

3. Barry F, Boynton RE, Liu B, Murphy JM. Chondrogenic differentiation of mesenchymal stem cells from bone marrow: differentiation-dependent gene expression of matrix components. Exp Cell Res 2001;268:189-200.

4. Ma HL, Hung SC, Lin SY, Chen YL, Lo WH. Chondrogenesis of human mesenchymal stem cells encapsulated in alginate beads. J Biomed Mater Res A 2003;64:273-81.

5. Cui JH, Park SR, Park K, Choi BH, Min BH. Preconditioning of mesenchymal stem cells with low-intensity ultrasound for cartilage formation in vivo. Tissue Eng 2007;13:351-60.

6. Huang CY, Hagar KL, Frost LE, Sun Y, Cheung HS. Effects of cyclic compressive loading on chondrogenesis of rabbit bonemarrow derived mesenchymal stem cells. Stem Cells 2004;22: 313-23.

7. Angele P, Yoo JU, Smith C, Mansour J, Jepsen KJ, Nerlich M, et al. Cyclic hydrostatic pressure enhances the chondrogenic phenotype of human mesenchymal progenitor cells differentiated in vitro. J Orthop Res 2003;21:451-7.

8. Indrawattana N, Chen G, Tadokoro M, Shann LH, Ohqushi H, Tateishi $\mathrm{T}$, et al. Growth factor combination for chondrogenic induction from human mesenchymal stem cell. Biochem Biophys Res Commun 2004;320:914-9.

9. Lee HJ, Choi BH, Min BH, Park SR. Low-intensity ultrasound stimulation enhances chondrogenic differentiation in alginate culture of mesenchymal stem cells. Artif Organs 2006;30:707-15.

10. Goldring MB, Tsuchimochi K, Ijiri K. The control of chondrogenesis. J Cell Biochem 2006;97:33-44.

11. Sekiya I, Vuoristo JT, Larson BL, Prockop DJ. In vitro cartilage formation by human adult stem cells from bone marrow stroma defines the sequence of cellular and molecular events during chondrogenesis. Proc Natl Acad Sci U S A 2002;99:4397-402.

12. Ylostalo J, Smith JR, Pochampally RR, Matz R, Sekiya I, Larson $\mathrm{BL}$, et al. Use of differentiating adult stem cells (marrow stromal cells) to identify new downstream target genes for transcription factors. Stem Cells 2006;24:642-52.

13. Yang JW, de Isla N, Huselstein C, Sarda-Kolopp MN, Li N, Li YP, et al. Evaluation of human MSCs cell cycle, viability and differentiation in micromass culture. Biorheology 2006;43:489-96.

14. Javazon EH, Colter DC, Schwarz EJ, Prockop DJ. Rat marrow stromal cells are more sensitive to plating density and expand more rapidly from single-cell-derived colonies than human marrow stromal cells. Stem Cells 2001;19:219-25.

15. Eslaminejad MB, Nikmahzar A, Taghiyar L, Nadri S, Massumi M. Murine mesenchymal stem cells isolated by low density primary culture system. Dev Growth Differ 2006;48:361-70.

16. Decheva D, Popov C, Mutschler W, Schieker M. Human mesenchymal stem cells in contact with their environment: surface characteristics and the integrin system. J Cell Mol Med 2007;11: 21-38.

17. Pittenger MF, Mackay AM, Beck SC, Jaiswal RK, Douglas R, Mosca JD, et al. Multilineage potential of adult human mesenchymal stem cells. Science 1999;284:143-7.

18. Wiesmann A, Buhring HJ, Mentrup C, Wiesmann HP. Decreased CD90 expression in human mesenchymal stem cells by applying mechanical stimulation. Head Face Med 2006;2:8.

19. Honczarenko M, Le Y, Swierkowski M, Ghiran I, Glodek AM, Silberstein LE. Human bone marrow stromal cells express a distinct set of biologically functional chemokine receptors. Stem Cells 2006;24:1030-41.

20. Diaz-Romero J, Gaillard JP, Grogan SP, Nesic D, Trub T, Mainil-Varlet P. Immunophenotypic analysis of human articular chondrocytes: changes in surface markers associated with cell expansion in monolayer culture. J Cell Physiol 2005;202:731-42. 
21. Park SR, Oreffo OC, Triffitt JT. Interconversion potential of cloned human marrow adipocytes in vitro. Bone 1999;24:549-54.

22. Kavalkovich KW, Boynton RE, Murphy JM, Barry F. Chondrogenic differentiation of human mesenchymal stem cells within an alginate layer culture system. In Vitro Cell Dev Biol Anim 2002;38:457-66.

23. Van Osch GJ, van der Veen SW, Burger EH, Verwoerd-Verhoef HL. Chondrogenic potential of in vitro multiplied rabbit perichondrium cells cultured in alginate beads in defined medium. Tissue Eng 2000;6:321-30.

24. Majumdar MK, Keane-Moore M, Buyaner D, Hardy WB, Moorman MA, McIntosh KR, et al. Characterization and functionality of cell surface molecules on human mesenchymal stem cells. J Biomed Sci 2003;10:228-41.

25. Grogan SP, Barbera A, Diaz-Romero J, Cleton-Jansen AM, Soeder S, Whiteside R, et al. Identification of markers to characterize and sort human articular chondrocytes with enhanced in vitro chondrogenic capacity. Arthritis Rheum 2007;56:586-95.

26. Mareschi K, Ferrero I, Rusticelli D, Aschero S, Gammaitoni L, Aglietta M, et al. Expansion of mesenchymal stem cells isolated from pediatric and adult donor bone marrow. J Cell Biochem 2006;97:744-54

27. Diaz-Romero J, Nesic D, Grogan SP, Heini P, Mainil-Varlet P. Immunophenotypic changes of human articular chondrocytes during monolayer culture reflect bona fide dedifferentiation rather than amplification of progenitor cells. J Cell Physiol 2008;214: $75-83$.

28. D'Ippolito G, Diabira S, Howard GA, Menei P, Roos BA, Schiller PC. Marrow-isolated adult multilineage inducible (MIAMI) cells, a unique population of postnatal young and old human cells with extensive expansion and differentiation potential. J Cell Sci 2004; 117(Pt 14):2971-81.

29. Summers KL, O’Donnell JL, Hoy MS, Peart M, Dekker J, Rothwell A, et al. Monocyte-macrophage antigen expression on chondrocytes. J Rheumatol 1995;22:1326-34.

30. Barbero A, Grogan SP, Mainil-Varlet P, Martin I. Expansion on specific substrates regulates the phenotype and differentiation capacity of human articular chondrocytes. J Cell Biochem 2006; 98:1140-9. 